\title{
MIMO Channel Capacity in 2D and 3D Isotropic Environments
}

\author{
Ryan J. Pirkl and Kate A. Remley \\ Electromagnetics Division, National Institute of Standards and Technology, RF Fields Group, 325 Broadway Street, MS 818.02, \\ Boulder, CO 80305, USA \\ Correspondence should be addressed to Ryan J. Pirkl, ryan.pirkl@nist.gov
}

Received 5 January 2012; Accepted 19 March 2012

Academic Editor: Markus Landmann

Copyright ( $) 2012$ R. J. Pirkl and K. A. Remley. This is an open access article distributed under the Creative Commons Attribution License, which permits unrestricted use, distribution, and reproduction in any medium, provided the original work is properly cited.

\begin{abstract}
We analyze theoretical distributions of MIMO channel capacity for different antennas in 2D and 3D statistically isotropic environments, which may be generated by multiprobe anechoic and reverberation chambers, respectively. We observe that the two environments yield comparable capacity distributions provided that (1) the 2D statistically isotropic environment's capacity data are taken at many different antenna orientations and (2) the radiation elements have a low directivity. When these conditions are met, we find that the relative error between the 2D statistically isotropic environment's orientation-combined capacity distribution and the $3 \mathrm{D}$ statistically isotropic environment's capacity distribution is typically less than $10 \%$ for signal-to-noise ratios greater than $5 \mathrm{~dB}$.
\end{abstract}

\section{Introduction}

Multiprobe anechoic chambers and reverberation chambers are capable of generating $2 \mathrm{D}$ and $3 \mathrm{D}$ statistically isotropic environments, respectively, suitable for over-the-air (OTA) testing of multiple-input multiple-output (MIMO) wireless terminals [1-3]. In CTIA, 3GPP's RAN4, and COST 2100 both multiprobe anechoic and reverberation chambers are being considered for adoption into certification test procedures for MIMO handsets $[1,4-6]$. It is possible that test procedures based on both chambers may be adopted, but it is unclear to what extent their test results are comparable. Here, we compare the statistically isotropic environments generated by multiprobe anechoic and reverberation chambers in terms of their capacity distributions for different MIMO antennas-under-test (AUTs).

Capacity determines the maximum obtainable throughput for a wireless device. The relationship between capacity and throughput implies that 2D and 3D statistically isotropic environments will only provide comparable device performance test results if their capacity statistics are comparable for any given AUT. Prior work has used channel simulations to compare the mean capacity of $2 \mathrm{D}$ and $3 \mathrm{D}$ statistically isotropic environments [2]. For a single orientation of the
AUT, large differences in the channel's mean capacity were observed for the 2D and 3D statistically isotropic environments. In contrast, by changing the orientation (and position) of the AUT between simulation trials, the mean capacities of the antennas converged to the same value in both environments.

We expand on the results presented in [2] by comparing the two environments' theoretical distributions of MIMO channel capacity for several AUTs. Our procedure extends the analysis presented in [7] to the problem of comparing capacity distributions for MIMO OTA test environments. We observe that for a single orientation of the AUT, the capacity distributions obtained from the two environments are unlikely to be comparable due to the orientation dependence of the $2 \mathrm{D}$ isotropic environment's capacity statistics. This is due to the well-known environment dependence of each AUT's complex correlation coefficient and mean effective gains (cf., [7-9]). We also observe that differences in the $2 \mathrm{D}$ and $3 \mathrm{D}$ environments' capacity distributions may be reduced substantially by comparing the $3 \mathrm{D}$ environment's orientationindependent capacity distribution to the 2D environment's orientation-combined capacity distribution, which is the distribution formed when capacity realizations are combined from different orientations of the AUT. However, we find 
that the difference between these two capacity distributions is small (e.g., within 10\%) only if the AUTs use lowdirectivity elements; for high-directivity elements, the difference between the capacity distributions may exceed $20 \%$. This suggests that discrepancies between device performance tests in statistically isotropic environments generated by multiprobe anechoic and reverberation chambers will be small provided that (1) the DUT's performance in the multiprobe anechoic chamber is evaluated at many different orientations, and (2) the DUT's dimensions are on the order of a wavelength or less such that the directivities of the antenna elements are low.

We begin in Section 2 by reviewing the covariance between the signals received by the elements of an AUT when it is placed in a random electromagnetic field. In Section 3, we define the power-angle spectra for 2D and 3D statistically isotropic environments, as well as the set of antenna configurations used in our numerical study of MIMO channel capacity. Capacity distributions for the AUTs in each test environment are presented and analyzed in Section 4. Section 5 explores why certain antenna configurations are more sensitive to orientation in 2D statistically isotropic environments than others. Section 6 summarizes the paper.

\section{Antennas in Random Electromagnetic Fields}

Let us consider a two-element antenna in a time-harmonic electromagnetic field. Assuming the antenna is in the farfield, whereby the electromagnetic field is composed entirely of homogeneous plane waves, we may express the signal $x_{i}$ received by the $i$ th antenna element as [8]

$$
x_{i}=\int d \Omega \mathbf{A}_{i}(\Omega) \cdot \mathbf{F}(\Omega),
$$

where $i \in\{1,2\}$, - denotes the vector dot product, $\mathbf{A}_{i}(\Omega)$ is the $i$ th element's complex vector radiation pattern defined as

$$
\mathbf{A}_{i}(\Omega)=A_{i h}(\Omega) \hat{\mathbf{h}}+A_{i v}(\Omega) \hat{\mathbf{v}}
$$

and $\mathbf{F}(\Omega)$ is the field's complex vector plane-wave spectrum defined as

$$
\mathbf{F}(\Omega)=F_{h}(\Omega) \hat{\mathbf{h}}+F_{v}(\Omega) \hat{\mathbf{v}}
$$

In (2) and (3), $\hat{\mathbf{h}}$ and $\hat{\mathbf{v}}$ are the two orthogonal unit vectors defining the horizontal and vertical polarizations, respectively, and $\Omega$ denotes a point on the unit sphere with $\int d \Omega$ corresponding to an integration over the unit sphere.

To constrain the scope of our capacity analysis, we consider power-normalized radiation patterns for which

$$
\int d \Omega\left\|\mathbf{A}_{i}(\Omega)\right\|^{2}=4 \pi
$$

That is, $\left\|\mathbf{A}_{i}(\Omega)\right\|^{2}$ is equal to the directivity of the $i$ th antenna element, where we neglect impedance mismatches and ohmic loss [8]. We recognize that mismatches and loss do affect the capacity of the MIMO wireless channel by way of a reduction in the signal-to-noise ratio (SNR) and, potentially, a gain imbalance at the receiver [10]. However, because impedance mismatches and ohmic losses are solely antennarelated quantities that are independent of the environment's power-angle spectrum, their effect on capacity does not provide insight into the differences between MIMO device tests in 2D and 3D statistically isotropic environments.

To describe a random electromagnetic field, we may specify the components, $F_{h}(\Omega)$ and $F_{v}(\Omega)$, of the planewave spectrum to be random variables characterized by the following covariances [11-13]:

$$
\begin{aligned}
& \mathcal{E}\left\{F_{h}(\Omega) F_{h}^{*}\left(\Omega^{\prime}\right)\right\}=P_{h}(\Omega) \delta\left(\Omega-\Omega^{\prime}\right), \\
& \mathcal{E}\left\{F_{v}(\Omega) F_{v}^{*}\left(\Omega^{\prime}\right)\right\}=P_{v}(\Omega) \delta\left(\Omega-\Omega^{\prime}\right), \\
& \mathcal{E}\left\{F_{h}(\Omega) F_{v}^{*}\left(\Omega^{\prime}\right)\right\}=\mathcal{E}\left\{F_{v}(\Omega) F_{h}^{*}\left(\Omega^{\prime}\right)\right\}=0,
\end{aligned}
$$

where $\mathscr{E}\{\cdot\}$ denotes the expectation operator, $\delta(\cdot)$ is the Dirac delta function, and $P_{h}(\Omega)$ and $P_{v}(\Omega)$ are real quantities that describe the power density versus angle-of-arrival for horizontally and vertically polarized incident plane waves, respectively. The electromagnetic field's vector power-angle spectrum is given by

$$
\mathbf{P}(\Omega)=P_{h}(\Omega) \hat{\mathbf{h}}+P_{v}(\Omega) \hat{\mathbf{v}}
$$

Denoting $P_{0}$ as the total power available to an AUT, we define the vector power-angle spectrum such that

$$
\int d \Omega[\mathbf{P}(\Omega) \cdot \hat{\mathbf{h}}+\mathbf{P}(\Omega) \cdot \hat{\mathbf{v}}]=P_{0} .
$$

The covariance between the signals received by the $i$ th and $j$ th antenna elements is defined as [13]

$$
\mathcal{E}\left\{x_{i} x_{j}^{*}\right\}=\rho_{i j} \sigma_{i} \sigma_{j},
$$

where $\sigma_{i}$ and $\sigma_{j}$ are the standard deviations of the signals received by $i$ th and $j$ th antenna elements, respectively, and $\rho_{i j}$ is the complex correlation coefficient of the two signals. Combining (1)-(8), the covariance may be written as

$$
\begin{aligned}
& \rho_{i j} \sigma_{i} \sigma_{j} \\
& \quad=\int d \Omega\left[A_{i h}(\Omega) A_{j h}^{*}(\Omega) P_{h}(\Omega)+A_{i v}(\Omega) A_{j v}^{*}(\Omega) P_{v}(\Omega)\right] .
\end{aligned}
$$

Interpreting $\sigma_{i}^{2}$ (the variance of $x_{i}$ ) as the power received by the $i$ th antenna element, we may define the element's mean effective gain (MEG) $\bar{g}_{i}$ as the ratio of received power to available power: $[8,14]$

$$
\bar{g}_{i}=\frac{\sigma_{i}^{2}}{P_{0}} .
$$

An element's MEG accounts for the interplay between the element's radiation pattern and the distribution of incident power versus angle-of-arrival. For an angle-invariant radiation pattern or power-angle spectrum, $\bar{g}_{i}=1 / 2$, indicating that one-half of the available power is received by the antenna 
TABLE 1: Specified antenna configurations.

\begin{tabular}{lcccccccc}
\hline Class & Configuration & \multicolumn{2}{c}{$2 \mathrm{D}$} & \multicolumn{2}{c}{$3 \mathrm{D}$} & $\max \left(\left\|\mathbf{A}_{i}(\Omega)\right\|^{2}\right)$ \\
\hline \multirow{3}{*}{$\begin{array}{l}\text { Closely spaced dipoles } \\
{[18]}\end{array}$} & $d=\lambda$ & 0.02 & 0.80 & 0.80 & 0.12 & 0.50 & 0.50 & 1.85 \\
& $d=\lambda / 2$ & 0.17 & 0.73 & 0.73 & 0.01 & 0.50 & 0.50 & 2.20 \\
& $d=\lambda / 4$ & 0.18 & 0.74 & 0.74 & 0.00 & 0.50 & 0.50 & 3.14 \\
\hline \multirow{2}{*}{$\begin{array}{l}\text { CTIA reference } \\
\text { antennas [19] }\end{array}$} & "Good" & 0.34 & 0.42 & 0.42 & 0.04 & 0.50 & 0.50 & 2.96 \\
& "Nominal" & 0.73 & 0.48 & 0.48 & 0.58 & 0.50 & 0.50 & 1.57 \\
\hline \multirow{2}{*}{$\begin{array}{l}\text { Cross-polarized } \\
\text { dipoles [20] }\end{array}$} & "Bad" & 0.90 & 0.54 & 0.54 & 0.90 & 0.50 & 0.50 & 1.77 \\
& Free space & 0.00 & 0.38 & 0.38 & 0.00 & 0.50 & 0.50 & 1.50 \\
\hline
\end{tabular}

[8]. By use of (8) and (9) with $i=j$ corresponding to the variance of $x_{i}$ (note that $\rho_{i i}=1$ ), we may express $\bar{g}_{i}$ in terms of the $i$ th antenna's vector radiation pattern and power-angle spectrum:

$$
\bar{g}_{i}=\frac{1}{P_{0}} \int d \Omega\left[\left|A_{i v}(\Omega)\right|^{2} P_{v}(\Omega)+\left|A_{i h}(\Omega)\right|^{2} P_{h}(\Omega)\right] .
$$

Finally, by combining (9)-(11), the environment-dependent correlation coefficient $\rho_{i j}$ may be expressed as

$$
\begin{aligned}
\rho_{i j}= & \frac{1}{P_{0} \sqrt{\bar{g}_{1} \bar{g}_{2}}} \\
& \times \int d \Omega\left[A_{i v}(\Omega) A_{j v}^{*}(\Omega) P_{v}(\Omega)+A_{i h}(\Omega) A_{j h}^{*}(\Omega) P_{h}(\Omega)\right] .
\end{aligned}
$$

\section{Simulation Setup}

In the following sections, we define the vector power-angle spectra and antenna configurations used in our numerical study of capacity in 2D and 3D statistically isotropic environments.

3.1. Power-Angle Spectra. For statistically isotropic environments, we expect that

$$
P_{h}(\Omega)=P_{v}(\Omega),
$$

whereby the power density versus angle-of-arrival is independent of the polarization of incident waves. For the 2D isotropic environment, the vector power-angle spectrum is only nonzero along the unit sphere's equator and is given by

$$
\mathbf{P}_{2 \mathrm{D}}(\Omega)=\frac{P_{0}}{2 \pi} \delta(\theta-\pi / 2) \frac{1}{2}(\hat{\mathbf{h}}+\hat{\mathbf{v}}),
$$

where $\theta$ denotes the zenith angle. In contrast, the 3D isotropic environment's vector power-angle spectrum is constant across the entire unit sphere and is given by

$$
\mathbf{P}_{3 \mathrm{D}}(\Omega)=\frac{P_{0}}{4 \pi} \frac{1}{2}(\hat{\mathbf{h}}+\hat{\mathbf{v}}) .
$$

Equations (14) and (15) are idealizations of the statistically isotropic environments that may be generated in multiprobe anechoic and reverberation chambers, respectively. Thus, we neglect non-idealities due to, for example, using a finite number of probes in the multiple-probe anechoic chamber [4-6] and unstirred energy in the reverberation chamber [15-17].

3.2. Antennas under Test. In the following sections, we will compare the capacity distributions for three classes of twoelement antennas.

(i) Two closely-spaced and vertically polarized Hertzian dipoles separated by a distance $d$ [18].

(ii) The recently developed CTIA MIMO reference antennas (so-called "Good", "Nominal", and "Bad") [19].

(iii) Two colocated and cross-polarized Hertzian dipoles aligned parallel to the $x-y$ plane and located (a) in free space and (b) at a height $h=\lambda / 4$ above a perfectly electrically conducting (PEC) surface with surface normal $\widehat{\mathbf{z}}[20]$.

The different antenna classes and configurations are intended to span the spectrum of antennas typically used by physically small handheld or mobile devices such as phones or laptops. Where applicable, our antenna descriptions include the effects of mutual coupling between elements.

Table 1 summarizes the different antenna configurations and compares the antennas' correlation coefficient magnitudes and MEGs for the 2D and 3D statistically isotropic environments as calculated by use of (11)-(15). Due to (4)'s power normalization, $\bar{g}_{i}=0.5$ for all antennas in the $3 \mathrm{D}$ statistically isotropic environment. For the crosspolarized dipoles above a PEC surface, $\bar{g}_{i}=0$ for the $2 \mathrm{D}$ statistically isotropic environment, because the gain patterns of the antenna's elements are zero for angles at and below the horizon [20]. The last column in Table 1 presents the maximum directivity of each antenna's elements. We will use these values later in Section 5. 


\section{Capacity}

We consider a 2-by-2 MIMO channel with uncorrelated transmit antennas and potentially correlated receive antennas. For the case of an uninformed transmitter and a receiver with perfect channel state information, the cumulative distribution function (CDF) of MIMO capacity is given by [21]

$$
\begin{aligned}
F_{C}(c)= & \sum_{i=1}^{2} \sum_{j=1, j \neq i}^{2} \frac{1}{\gamma_{i} \gamma_{j}\left(\gamma_{i}-\gamma_{j}\right)} \\
& \times\left\{e^{1 / \gamma_{i}+1 / \gamma_{j}} \frac{\gamma_{j}-\gamma_{i}}{\gamma_{i}}\left(\int_{1}^{2^{c}} d v v e^{-v / \gamma_{i}-2^{c} / v \gamma_{j}}\right)\right. \\
& \left.+\left[\gamma_{i}+\left(\gamma_{i}-\gamma_{j}\right)\left(\gamma_{i}+2^{c}\right)\right] e^{\left(1-2^{c}\right) / \gamma_{i}}\right\},
\end{aligned}
$$

where $\gamma_{i}$ are the eigenvalues of the 2 -by-2 matrix $\Gamma$ defined as

$$
\Gamma=\frac{1}{N_{0} n_{t}} \boldsymbol{\Sigma}_{r}
$$

In (17), $n_{t}=2$ is the number of transmit antenna elements, $N_{0}$ is the noise power density at the receiver, and $\Sigma_{r}$ is the receiver's covariance matrix given by

$$
\Sigma_{r}=\left[\begin{array}{ll}
\mathcal{E}\left\{\left|x_{1}\right|^{2}\right\} & \mathcal{E}\left\{x_{1} x_{2}^{*}\right\} \\
\mathcal{E}\left\{x_{2} x_{1}^{*}\right\} & \mathcal{E}\left\{\left|x_{2}\right|^{2}\right\}
\end{array}\right] .
$$

Using (8)-(12), $\boldsymbol{\Sigma}_{r}$ may be expressed as

$$
\boldsymbol{\Sigma}_{r}=P_{0}\left[\begin{array}{cc}
\bar{g}_{1} & \rho \sqrt{\bar{g}_{1} \bar{g}_{2}} \\
\rho^{*} \sqrt{\bar{g}_{1} \bar{g}_{2}} & \bar{g}_{2}
\end{array}\right],
$$

where the eigenvalues of $\boldsymbol{\Gamma}$ are

$$
\gamma_{2}^{1}=\frac{P_{0}}{N_{0} n_{t}}\left[\frac{\bar{g}_{1}+\bar{g}_{2} \pm \sqrt{\bar{g}_{1}^{2}+\bar{g}_{2}^{2}+2 \bar{g}_{1} \bar{g}_{2}\left(2|\rho|^{2}-1\right)}}{2}\right],
$$

and $\left(P_{0} / 2\right) / N_{0}$ defines the SNR of the corresponding singleinput single-output link for an isotropic antenna.

4.1. Single Orientation. Figures $1(\mathrm{a})$ and 1(b) compare the analytic 2-by-2 MIMO capacity CDFs for the different AUTs in the 2D and 3D statistically isotropic environments, respectively. The CDFs were calculated from (16)-(20) with the correlation coefficients and MEGs reported in Table 1. We observe that the different test environments may yield considerably different capacity CDFs for identical AUTs. The cross-polarized dipoles above a PEC surface are particularly problematic, because the MEGs of the antenna elements are zero (see Table 1) for the 2D environment. This implies an SNR of zero and results in a capacity CDF given by a unit step function with a transition at zero bps/Hz.

Figure 1(c) illustrates the shift in the capacity CDFs for the $2 \mathrm{D}$ statistically isotropic environment with respect to the
3D statistically isotropic environment. We quantify this shift in terms of a relative error of the 2D environment's capacity curves with respect to the $3 \mathrm{D}$ environment's capacity CDFs. We note that the relative error for the cross-polarized dipoles above a PEC surface is $100 \%$ due to its step-function capacity $\mathrm{CDF}$ in the $2 \mathrm{D}$ isotropic environment; this error is outside of the range of relative errors considered in Figure 1(c). Depending on the antenna configuration, the relative error in the 2D environment's capacity CDF may exceed $20 \%$. This suggests that capacity statistics for a given AUT in a $2 \mathrm{D}$ and $3 \mathrm{D}$ statistically isotropic environment may not be comparable for a single orientation of the AUT. This is in agreement with the conclusions presented in [2] for ergodic capacity based on a single orientation of the AUT.

4.2. Multiple Orientations. As suggested in [2], a possible solution for obtaining comparable capacity statistics is to combine capacity data from different orientations of the AUT in the 2D statistically isotropic environment. Assuming $N$ orientations of the AUT are sampled equally (i.e., an equal number of independent capacity realizations are taken at $N$ unique orientations), the CDF of the combined set of capacity realizations is equal to the mean of the $N$ orientation-dependent capacity CDFs: [22]

$$
F_{\hat{C}}(c)=\frac{1}{N} \sum_{n=1}^{N} F_{C}^{(n)}(c),
$$

where $F_{C}^{(n)}(c)$ denotes the capacity CDF associated with the $n$th orientation of the AUT, and $F_{\hat{C}}(c)$ describes the orientation-combined CDF of the resulting (mixture) distribution.

As Figure 2 illustrates, there are three degrees-of-freedom in a $3 \mathrm{D}$ rotation of an object to a new orientation [23-25]. In the rotation sequence illustrated in Figure 2, an object is rotated by $\alpha \in[0, \pi)$ in a direction $\beta \in[0,2 \pi)$ such that object's original $z$-axis is aligned to an axis $z^{\prime}$ defined by $(\alpha, \beta)$. Then, the object is rotated by an angle $\chi \in[0,2 \pi)$ about the $z^{\prime}$-axis. To obtain a uniform sampling of the AUT's $3 \mathrm{D}$ orientation space, we apply the successive orthogonal images technique [24]. We specify a set of $L$ angle pairs $(\alpha, \beta)$ corresponding to $L$ points uniformly distributed on the unit sphere. For each angle pair, $\chi=2 \pi(m / M)$ for $m=$ $0,1, \ldots, M-1$, where $M$ is an integer number of $\chi$ rotations per $(\alpha, \beta)$ pair as given by

$$
M=\operatorname{round}(\sqrt{\pi L}) \text {, }
$$

and the total number of orientations is $N=M L$. In (22), round $(\cdot)$ rounds to the nearest integer. Equation (22) provides the appropriate weighting between $M$ and $L$ to ensure that the $N$ orientations are a uniform sampling of the rotation space (cf., [24, Equation (3.1)]).

A set of $L=162$ uniformly distributed angle pairs $(\alpha, \beta)$ were generated by iterative triangularization of an icosahedron. By use of (22), we have $M=23$ whereby a set of $N=M L=3726$ uniformly distributed orientations were considered. (Similar results were observed for larger 


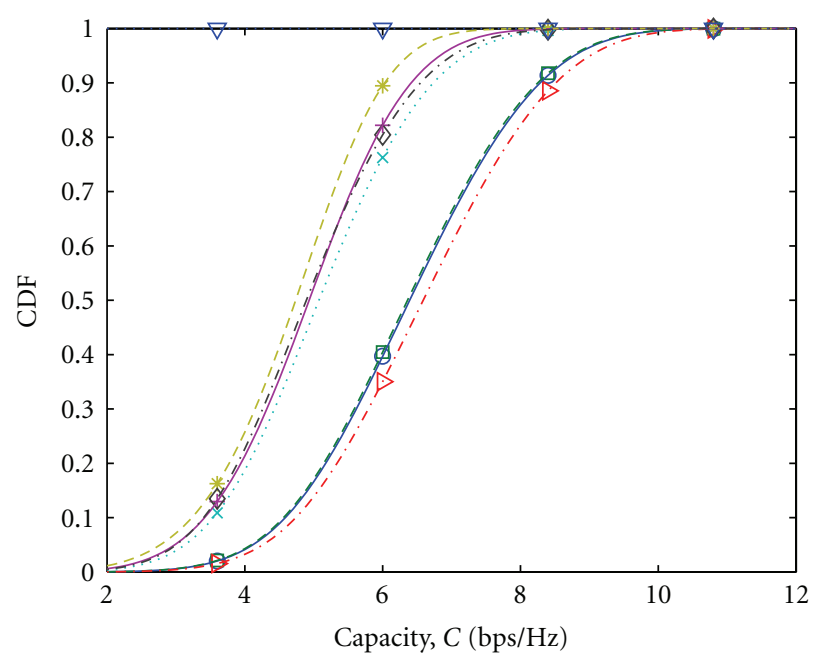

(a) $2 \mathrm{D}$ environment

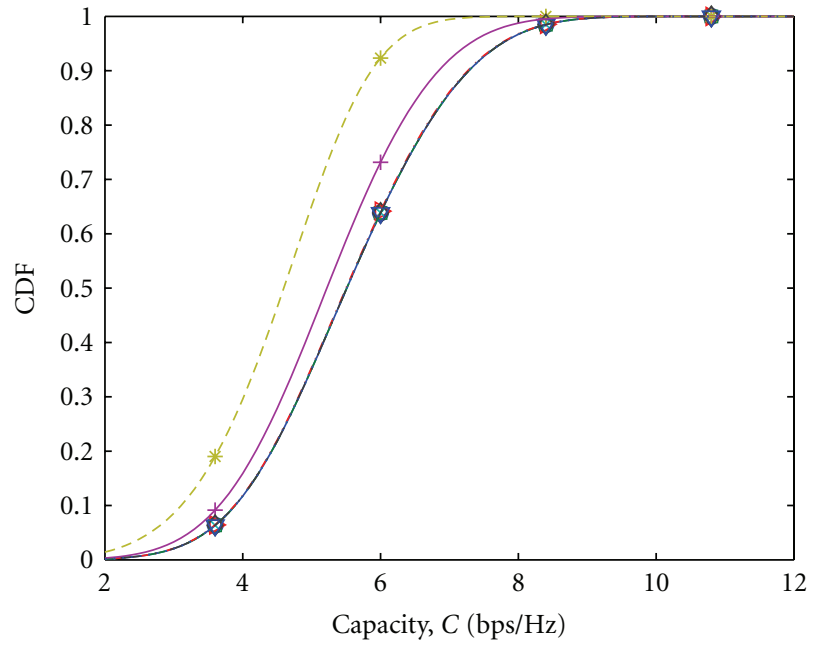

(b) $3 \mathrm{D}$ environment

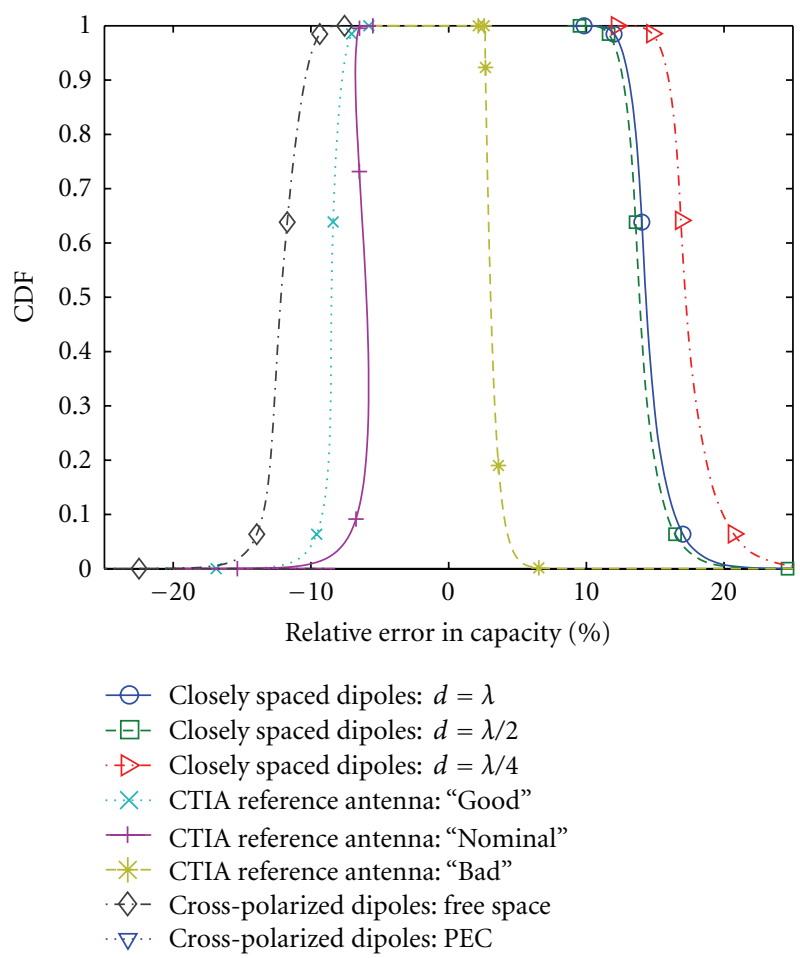

(c) relative error

FIGURE 1: Comparison of the capacity CDFs for the different AUTs in statistically isotropic environments at a single orientation: (a) capacity CDFs for the $2 \mathrm{D}$ environment, (b) capacity CDFs for the 3D environment, (c) relative error in the 2D environment's capacity CDFs with respect to the $3 \mathrm{D}$ environment's capacity CDFs. The center plot's legend applies to all three plots.

choices of $N$.) The AUT was rotated to each of the $N$ orientations, and (11), (12), and (16) were used to calculate the resulting $N$ capacity CDFs. For each of the AUTs, Figure 3 compares the $N$ orientation-dependent capacity CDFs obtained from the 2D statistically isotropic environment (solid lines), the resulting orientation-combined CDF from the 2D statistically isotropic environment (solid line), and the orientation-independent capacity CDF obtained from the 3D statistically isotropic environment (dashed line). We observe large variations in the $2 \mathrm{D}$ environment's orientationdependent capacity CDFs for the closely spaced dipoles (Figures 3(a)-3(c)), as well as the cross-polarized dipoles above a PEC surface (Figure 3(h)).

Figures 4(a) and 4(b) compare the 2D environment's orientation-combined capacity $\mathrm{CDFs}$ to those from the $3 \mathrm{D}$ environment's orientation-independent capacity CDFs; Figure 4(c) examines the relative error in the 2D statistically isotropic environment's orientation-combined capacity 


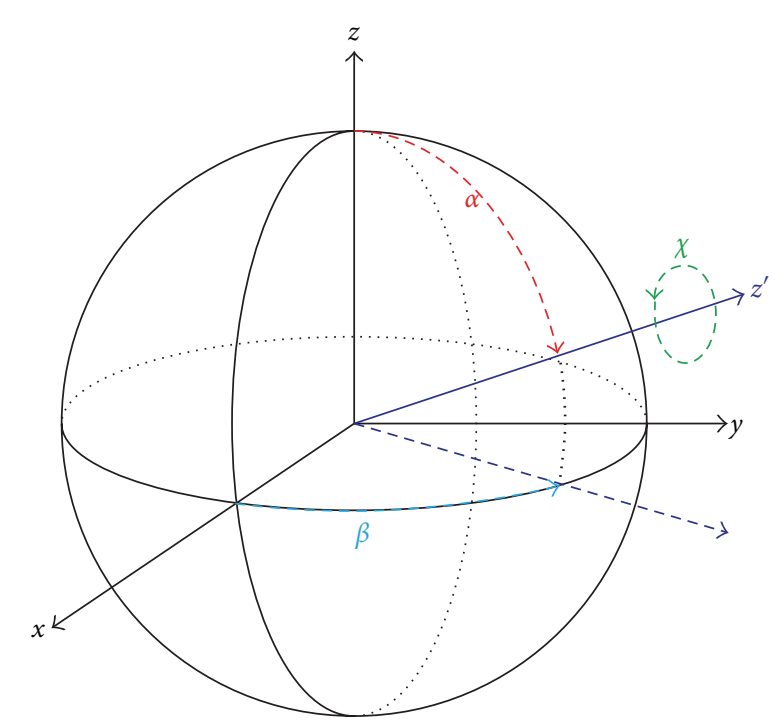

Figure 2: There are three degrees of freedom in the specification of a rotation to an arbitrary orientation. The diagram defines a rotation of an object in $3 \mathrm{D}$ via Euler angles. The object is rotated by an angle $\alpha$ in the direction $\beta$ and subsequently rotated by an angle $\chi$ about the axis $z^{\prime}$ defined by $(\alpha, \beta)$.

CDFs with respect to the 3D statistically isotropic environment's orientation-independent capacity CDFs. We observe that for the CTIA reference antennas and cross-polarized dipoles in free space, this "error" is quite small, with typical magnitudes below 2\%. In comparison, the relative error of the cross-polarized dipoles above the PEC surface and, to an extent, the closely-spaced dipoles, is much larger, with magnitudes exceeding 5-20\%. Note that these are the same antenna configurations which showed the greatest variation in the 2D environment's orientation-dependent capacity CDFs (see Figure 3). Thus, Figures 3 and 4(c) suggest that for a given AUT, the 2D environment's orientation-combined capacity statistics will be comparable to the $3 \mathrm{D}$ environment's orientation-independent capacity statistics provided that the variations in the $2 \mathrm{D}$ environment's orientationdependent capacity CDFs are small. Repeat studies at SNRs of $5 \mathrm{~dB}$ to $20 \mathrm{~dB}$ yielded similar errors, albeit with a slight increase in relative error for decreasing SNR. The typical range of error magnitudes remained below $10 \%$.

\section{Sensitivity of Capacity to AUT Orientation in 2D Statistically Isotropic Environments}

From Figures 3 and 4(c), we observed that for a given AUT, large orientation-dependent variations in the 2D environment's capacity CDF led to a large relative error between the $2 \mathrm{D}$ environment's orientation-combined capacity CDF and the 3D environment's orientation-independent capacity CDF. Here, we investigate the source of these large orientation-dependent variations in capacity and determine why some antennas are more sensitive to orientation in the $2 \mathrm{D}$ isotropic environment than others. This provides insight into the range of antenna configurations for which we may expect the 2D environment's orientation-combined capacity CDF to be comparable to the $3 \mathrm{D}$ environment's orientationindependent capacity CDF. We base our analysis on the orientation-dependence of each AUT's correlation coefficient and MEGs in the 2D statistically isotropic environment. Note that for the 3D environment, these quantities are orientation invariant and are tabulated in Table 1.

Figure 5 presents scatter plots of each AUT's MEGs and correlation coefficient for different orientations in the $2 \mathrm{D}$ statistically isotropic environment. The location of each circle corresponds to the MEG of the AUT's elements; the circle's size/shade corresponds to the magnitude of the correlation coefficient. We observe that the antennas exhibiting the largest variations in the 2D environment's orientation-dependent capacity CDFs, namely, the cross-polarized dipoles above a PEC surface and the closely-spaced dipoles tend to have similar antenna element MEGs at each orientation (i.e., $\bar{g}_{1} \approx \bar{g}_{2}$ ). This indicates that the average power received by each of the antenna elements is identical, whereby a low (or high) average received power at one element implies an equally low (or high) average received power at the other element. This allows for large variations in the cumulative power received by the two antenna elements. In contrast, the AUTs exhibiting the smallest variations in the orientationdependent capacity CDFs tend to have MEGs that follow $\bar{g}_{1}+$ $\bar{g}_{2} \approx 1$. This indicates that the cumulative power received by both antenna elements is approximately constant at different orientations, whereby a low average received power at one element is compensated by a high average received power at the other element.

Based on Figure 5, the 2D environment's orientationdependent capacity CDFs appear to be more sensitive to joint orientation-dependent variations in the MEGs of an AUT's elements than orientation-dependent variations in its correlation coefficient magnitude (e.g., compare Figures 5(g), 5(h), 3(g) and 3(h)). To confirm this, Figure 6 presents environment-independent contour plots of the median capacity for different correlation coefficient magnitudes and MEGs. For the calculations, we specify a nominal single-input single-output SNR of $10 \mathrm{~dB}$ (i.e., $\left.\left(P_{0} / 2\right) / N_{0}=10\right)$. Figure $6(\mathrm{a})$ corresponds to the case of equal antenna element MEGs; Figure 6(b) corresponds to the case of constant cumulative received power. As indicated by the range of median capacities for the two cases, the equal MEG case $\left(\bar{g}_{1}=\bar{g}_{2}\right)$ allows for larger variations in the median capacity than the constant cumulative received power case $\left(\bar{g}_{1}+\bar{g}_{2}=1\right)$. We also observe for both cases that the effect on capacity of large variations in the magnitude of the correlation coefficient is considerably weaker than large variations in MEG. This indicated by the smaller gradient for changes in $\bar{g}_{1}$ as compared to changes in $\left|\rho_{12}\right|$.

Based on Figures 3-6 and the maximum directivities listed in Table 1, we expect that the 2D environment's orientation-combined capacity statistics should be comparable to the 3D environment's orientation-independent capacity statistics provided that the maximum directivities of the AUT's elements are small (e.g., $\max \left(\left\|\mathbf{A}_{i}(\Omega)\right\|^{2}\right) \leq 2$ ). This criterion should lead to smaller orientation-dependent 


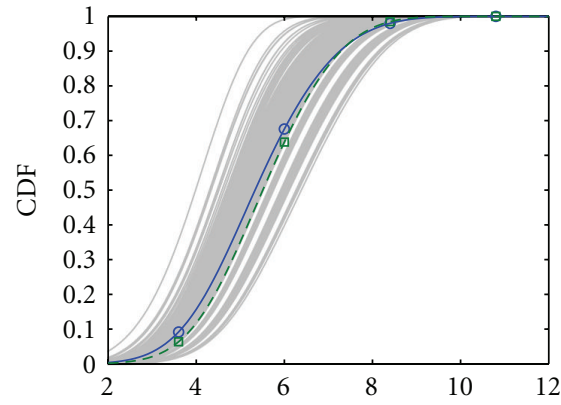

(a) Closely spaced dipoles: $d=\lambda$

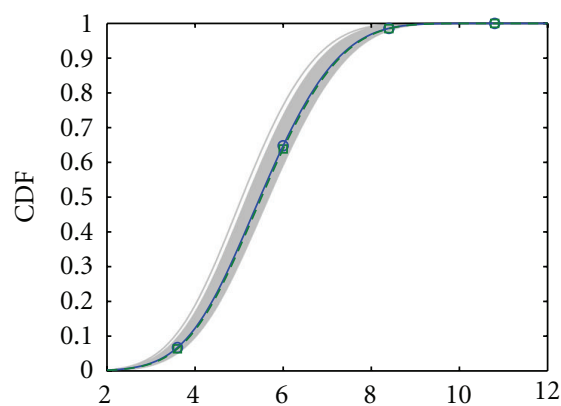

(d) CTIA reference antenna: "Good"

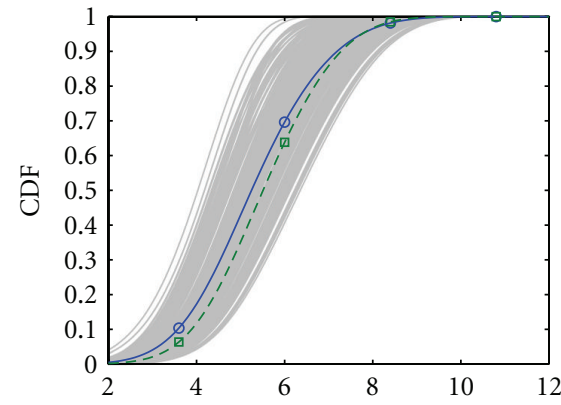

(b) Closely spaced dipoles: $d=\lambda / 2$

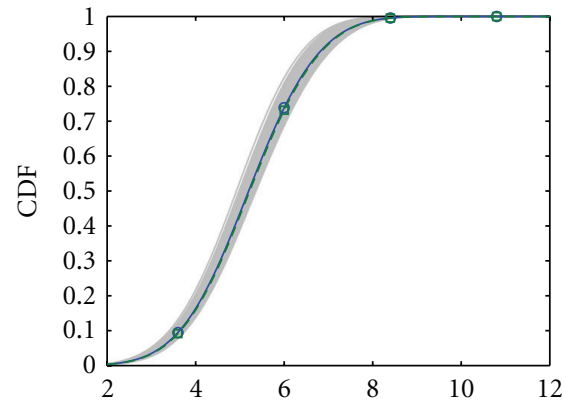

(e) CTIA reference antenna: "Nominal"

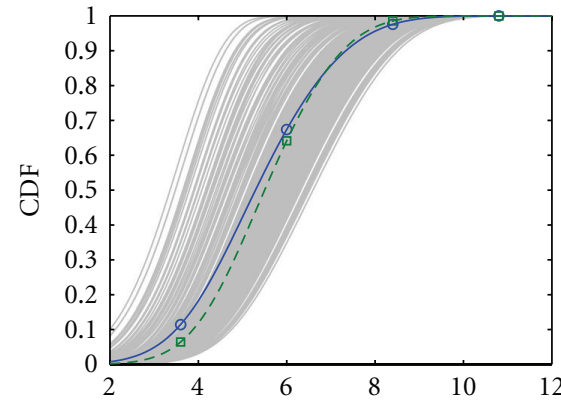

(c) Closely spaced dipoles: $d=\lambda / 4$

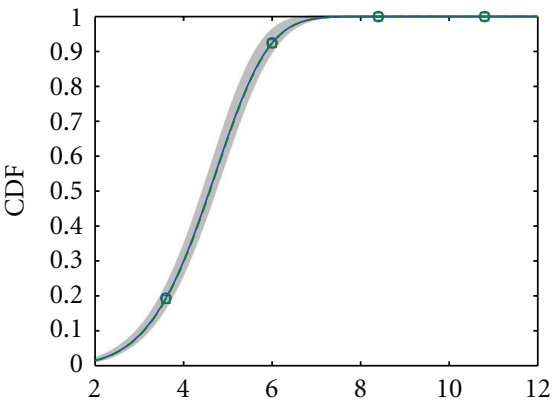

(f) CTIA reference antenna: "Bad"

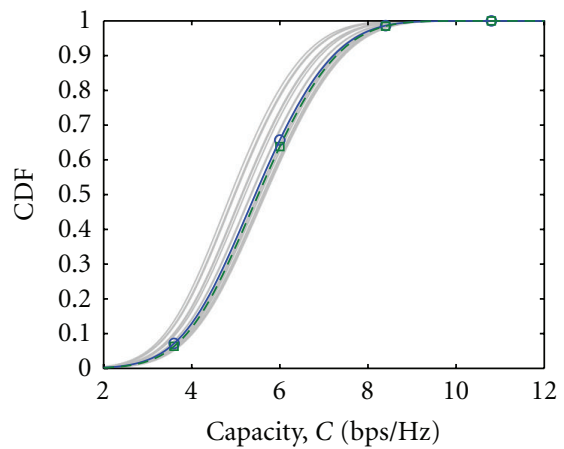

- 2D orientation dependent

$\rightarrow 2 \mathrm{D}$ orientation combined

$-\sharp-3 \mathrm{D}$ orientation independent

(g) Cross-polarized dipoles: free space

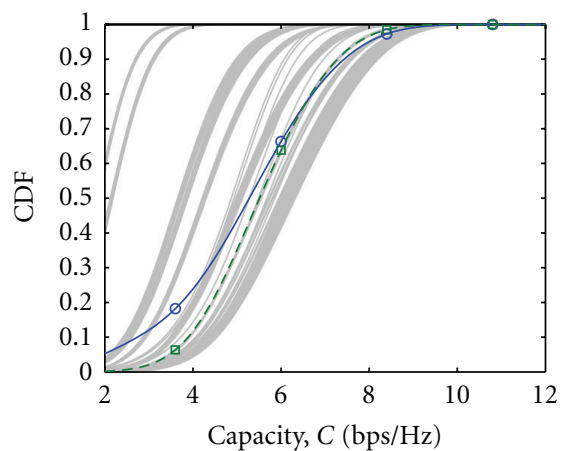

- 2D orientation dependent

$\rightarrow 2 \mathrm{D}$ orientation combined

$-\bullet-3 \mathrm{D}$ orientation independent

(h) Cross-polarized dipoles: PEC

FIGURE 3: Comparison of the capacity CDFs for multiple orientations of the two-element antennas in statistically isotropic environments. Each plot compares the 2D environment's orientation-dependent capacity CDFs, the 2D environment's orientation-combined capacity CDFs, and the 3D environment's orientation-independent capacity CDFs.

fluctuations in the AUT's MEGs and, thereby, cumulative received power. In $[7,14]$, we note that similar conclusions were reached concerning the greater variability of MEG for different orientations of antennas with large directivities. Here, we have demonstrated that the variability in capacity is dominated by this variability in MEG. Antennas with markedly different element directivities may also yield comparable capacity CDFs, because it is less likely that $\bar{g}_{1} \approx$ $\bar{g}_{2}$. However, based on the antenna configurations considered here, we expect that low directivity is the more important criteria.

\section{Conclusions}

Channel capacity determines the maximum theoretical throughput for a given wireless link. By designing test procedures such that the capacity distributions obtained for a given AUT in a 2D and 3D statistically isotropic environment are comparable, we expect that throughput statistics for a corresponding DUT in these two environments will likewise be comparable. More so, we expect that discrepancies between observed throughput statistics for different devices in different test environments will be largely 


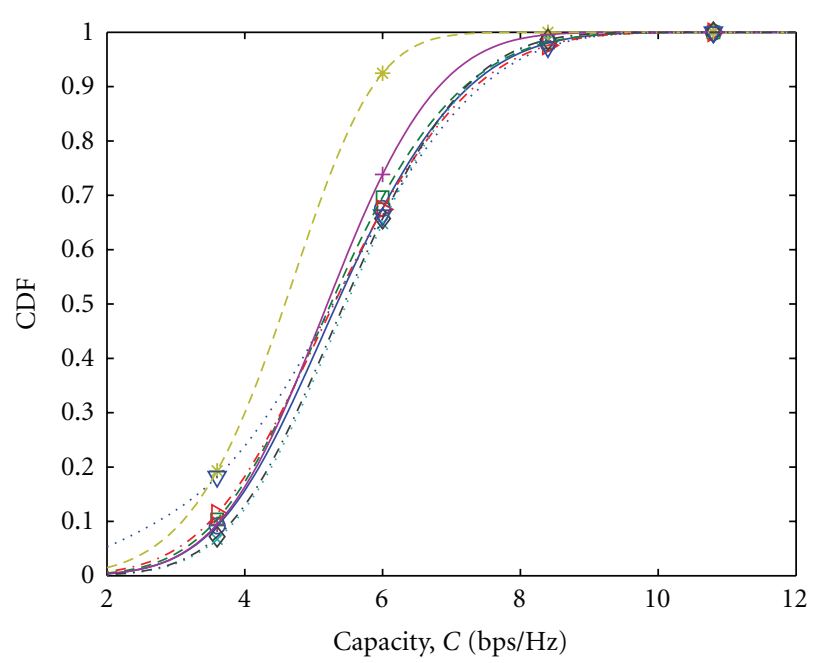

(a) $2 \mathrm{D}$ environment

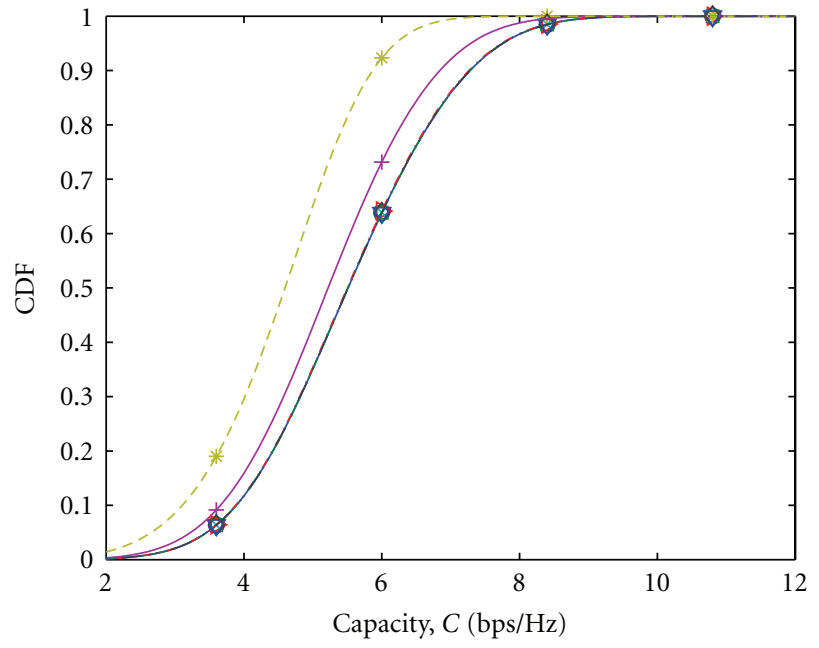

(b) 3D environment

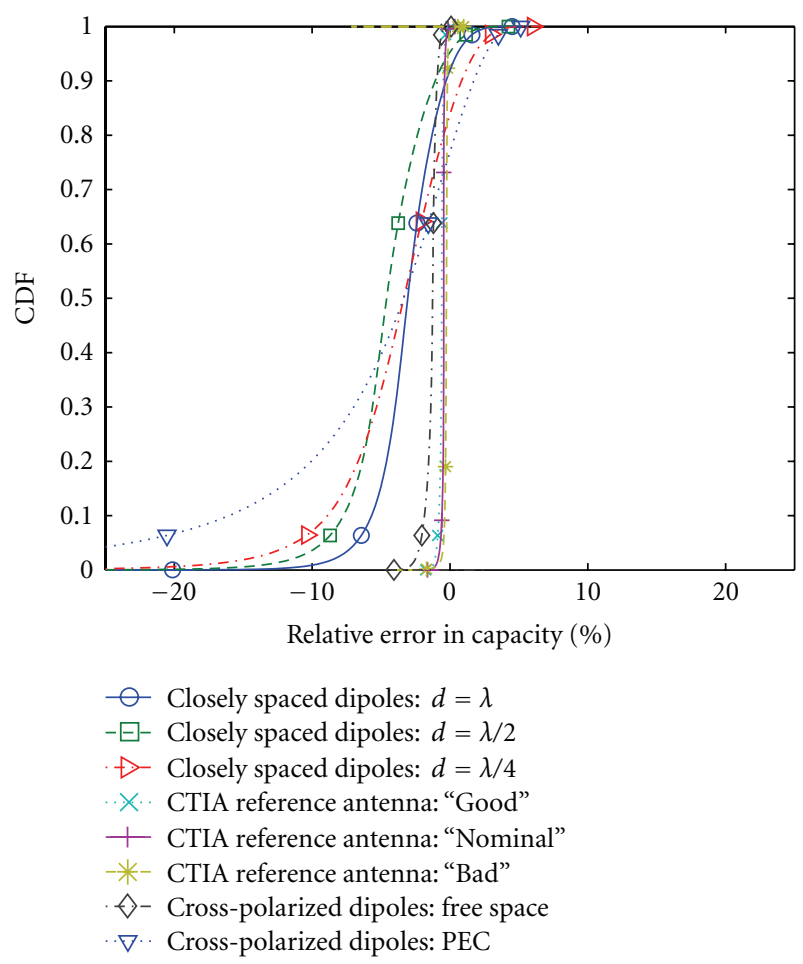

(c) Relative error

FIgure 4: Comparison of the capacity CDFs for the different AUTs in statistically isotropic environments at multiple orientations: (a) orientation-combined capacity CDFs for the 2D environment, (b) orientation-independent capacity CDFs for the 3D environment, (c) relative error in the $2 \mathrm{D}$ environment's orientation-combined capacity CDFs with respect to the $3 \mathrm{D}$ environment's orientation-independent capacity CDFs. The center plot's legend applies to all three plots.

due to fundamental differences in the devices (e.g., antenna loss and mismatch, receiver sensitivity, and algorithm implementation) and not fundamental differences in the capacity statistics of the test environments, assuming a sufficient number of AUT orientations in the 2D environment.

Based on the analysis presented here, provided that (1) the DUT is rotated to different orientations in the $2 \mathrm{D}$ statistically isotropic environment, and (2) the dimensions of the DUT are on the order of a wavelength or less such that the element directivities will be low, we expect that throughput statistics for a DUT in 2D and 3D statistically isotropic environments will be within $10 \%$ of each other, as suggested by Figures 3 and 4(c). This suggests that with properly designed test procedures, over-the-air tests of multiantenna wireless terminals in multiprobe anechoic and reverberation chambers should be comparable for a 


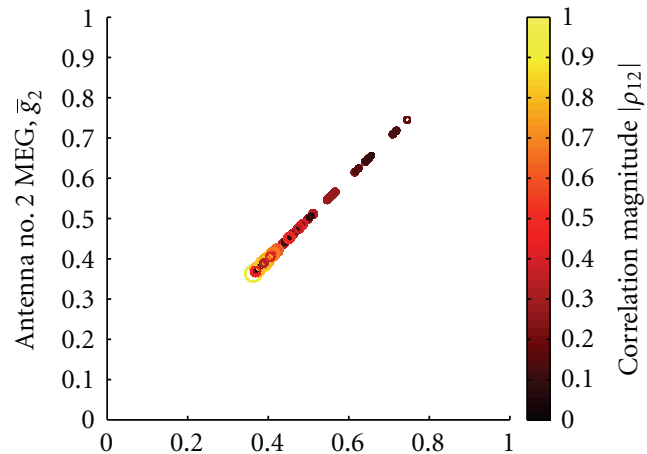

(a) closely-spaced dipoles: $d=\lambda$

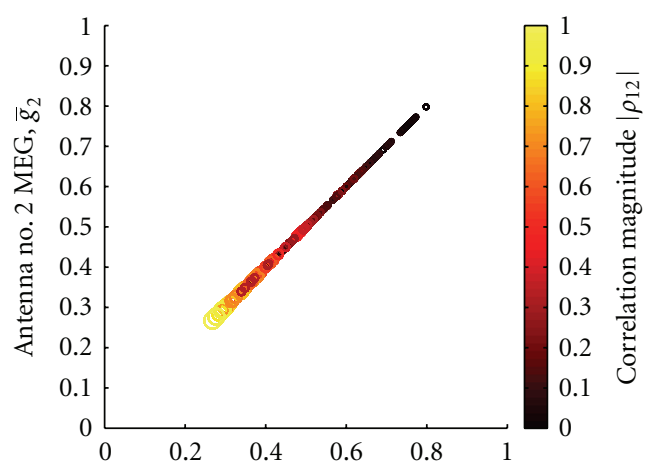

(c) Closely spaced dipoles: $d=\lambda / 4$

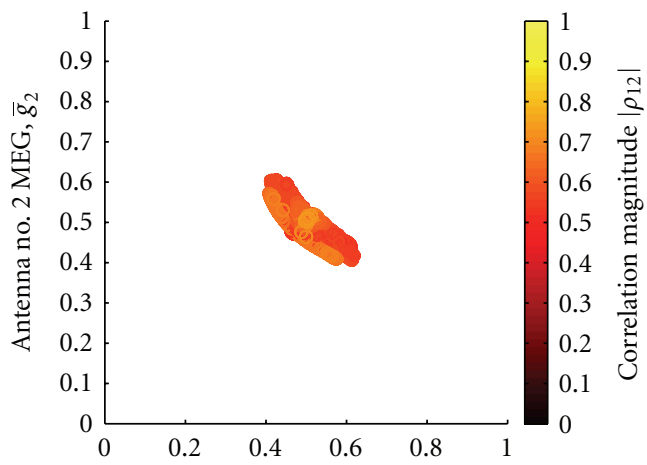

(e) CTIA reference antenna: "Nominal"

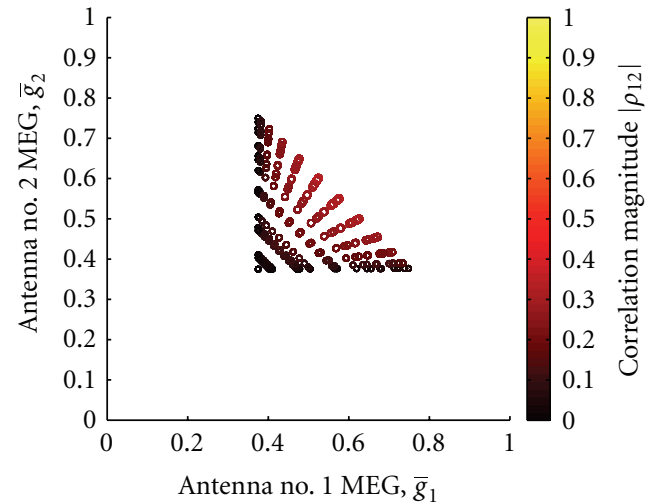

(g) Cross-polarized dipoles: free space

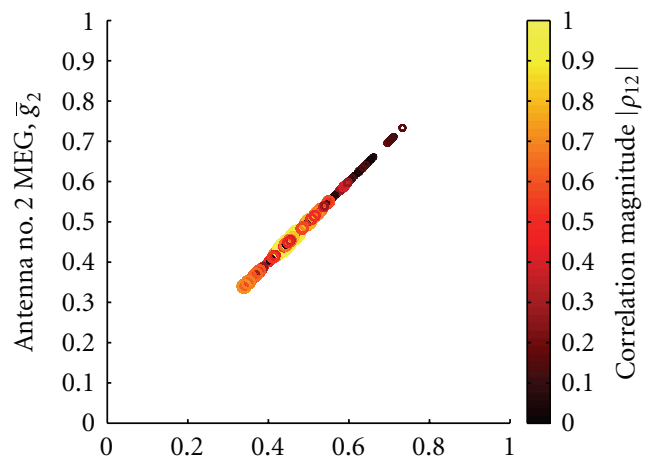

(b) Closely spaced dipoles: $d=\lambda / 2$

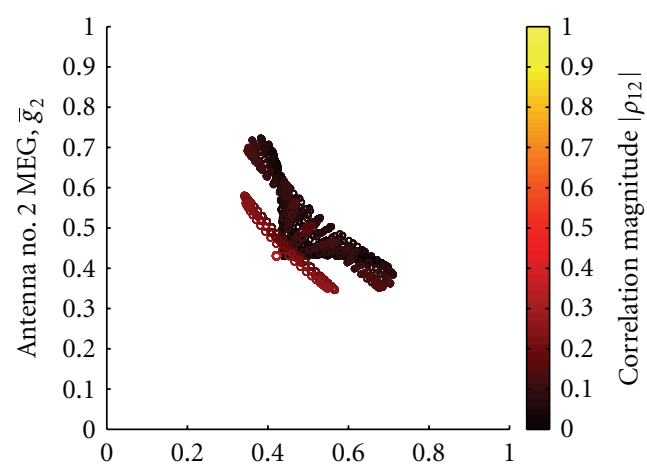

(d) CTIA reference antenna: "Good"

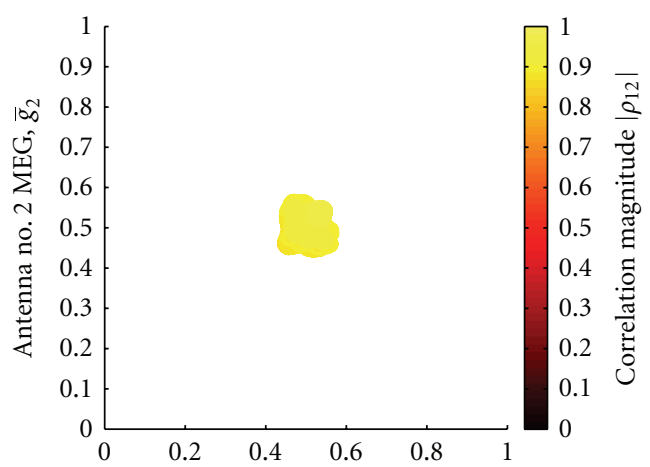

(f) CTIA reference antenna: "Bad"

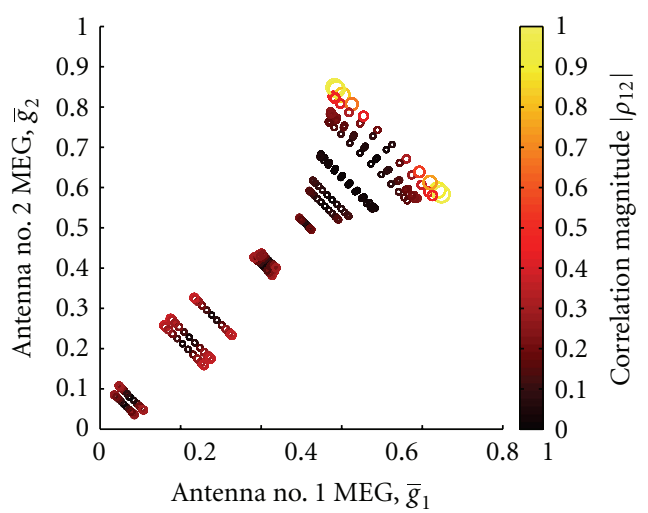

(h) Cross-polarized dipoles: PEC

FIGURE 5: Scatter plots of mean effective gain (MEG) and correlation coefficient magnitudes for each AUT in the 2D statistically isotropic environment for $N$ uniformly distributed orientations of the AUT. The position of each circle indicates the MEGs of the two antenna elements; the size/shade of each circle indicates the magnitude of the correlation coefficient. 


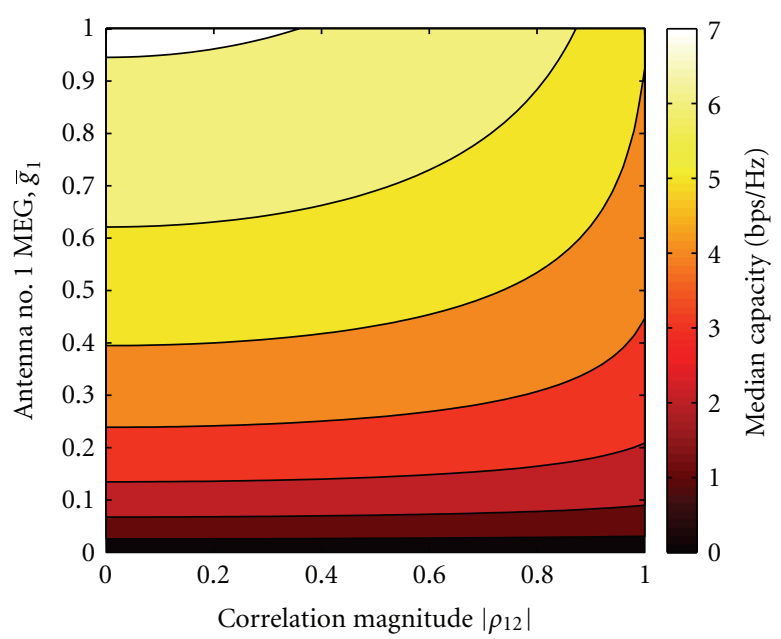

(a) $\bar{g}_{2}=\bar{g}_{1}$

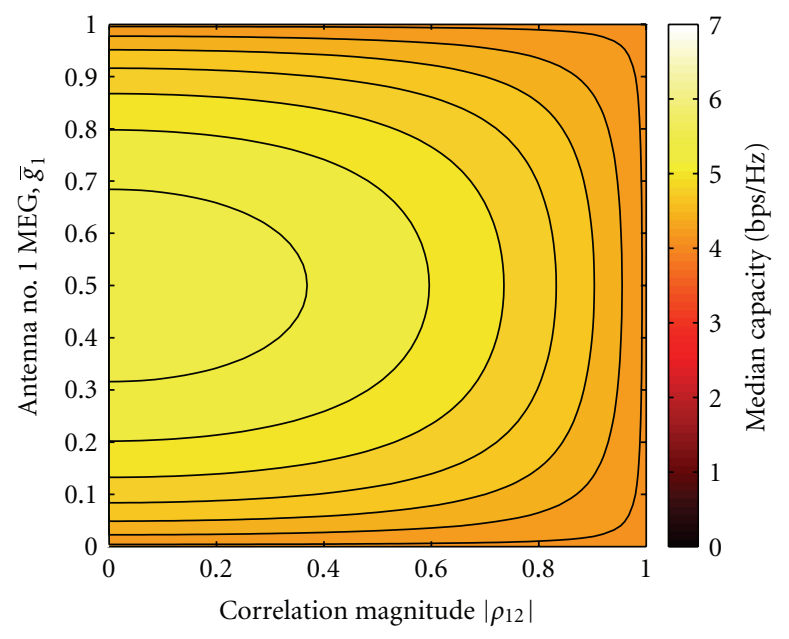

(b) $\bar{g}_{2}=1-\bar{g}_{1}$

FIGURE 6: Median capacity for ranges of correlation coefficient magnitudes $\left|\rho_{12}\right|$ and MEGs, $\bar{g}_{1}$ and $\bar{g}_{2}$.

wide range of devices. Finally, it is worth emphasizing that our conclusions are based solely on the distribution of capacity. That is, our conclusions are independent of the actual number of realizations used to calculate a capacity (or throughput) statistic and apply even for the case where a single capacity (or throughput) measurement is made at each orientation.

\section{Acknowledgments}

The authors acknowledge the cochair of CTIA's MIMO OTA Subgroup (MOSG), Scott Prather of AT\&T Wireless, and the lead designer of the the CTIA MIMO reference antennas, Istvan Szini of Motorola Mobility, for providing the complex radiation pattern data for the CTIA MIMO reference antennas. This work was supported by a National Research Council Postdoctoral Research Associateship.

\section{References}

[1] 3rd Generation Partnership Project; Technical Specification Group Radio Access Networks; Measurement of radiated performance for MIMO and multi-antenna reception for HSPA and LTE terminals (Release 10), 3GPP Std. TR 37.976, Rev. 1.4.0, 2009.

[2] U. Carlberg, J. Carlsson, A. Hussain, and P.-S. Kildal, "Ray based multipath simulation tool for studying convergence and estimating ergodic capacity and diversity gain for antennas with given far-field functions," in Proceedings of the International Conference on Applied Electromagnetics and Communications (ICECom '10), pp. 1-4, Dubrovnik, Croatia, September 2010.

[3] J. Carlsson, U. Carlberg, A. Hussain, and P.-S. Kildal, "About measurements in reverberation chamber and isotropic reference environment," in Proceedings of the International Conference on Applied Electromagnetics and Communications (ICECom '10), pp. 1-4, Dubrovnik, Croatia, September 2010.

[4] J. P. Nuutinen, P. Kyösti, Y. Gao, and M. D. Foegelle, "On the MIMO OTA test system," in Proceedings of the 5th International
ICST Conference on Communications and Networking in China (ChinaCom '10), pp. 1-5, Beijing, China, August 2010.

[5] T. Laitinen, P. Kyösti, J.-P. Nuutinen, and P. Vainikainen, "On the number of OTA antenna elements for plane-wave synthesis in a MIMOOTA test system involving a circular antenna array," in Proceedings of the European Conference on Antennas and Propagation, pp. 1-5, Barcelona, Spain, April 2010.

[6] T. Laitinen, J. Toivanen, P. Kyösti, J.-P. Nuutinen, and P. Vainikainen, "On a MIMO-OTA testing based on multi-probe technology," in Proceedings of the URSI International Symposium on Electromagnetic Theory, pp. 227-230, Berlin, Germany, August 2010.

[7] B. Yanakiev, J. Ø. Nielsen, M. Christensen, and G. F. Pedersen, "Antennas in real environments," in Proceedings of the 5th European Conference on Antennas and Propagation (EUCAP '11), pp. 2766-2770, Rome, Italy, April 2011.

[8] R. Vaughan and J. B. Andersen, Channels, Propagation and Antennas for Mobile Communications, The Institution of Electrical Engineers, London, UK, 2003.

[9] B. Yanakiev, J. Nielsen, M. Christensen, and G. F. Pedersen, "On small terminal antenna correlation and impact on MIMO channel capacity," IEEE Transactions on Antennas and Propagation, vol. 60, no. 2, pp. 689-699, 2010.

[10] K. Ogawa, S. Amari, H. Iwai, and A. Yamamoto, "Effects of received power imbalance on the channel capacity of a handset MIMO," in Proceedings of the IEEE International Symposium on Personal, Indoor and Mobile Radio Communications (PIMRC '07), pp. 1-5, Athens, Greece, September 2007.

[11] D. A. Hill, "Plane wave integral representation for fields in reverberation chambers," IEEE Transactions on Electromagnetic Compatibility, vol. 40, no. 3, pp. 209-217, 1998.

[12] D. A. Hill and J. M. Ladbury, "Spatial-correlation functions of fields and energy density in a reverberation chamber," IEEE Transactions on Electromagnetic Compatibility, vol. 44, no. 1, pp. 95-101, 2002.

[13] P. De Doncker and R. Meys, "Statistical response of antennas under uncorrelated plane wave spectrum illumination," Electromagnetics, vol. 24, no. 6, pp. 409-423, 2004.

[14] A. Alayón Glazunov, A. F. Molisch, and F. Tufvesson, "Mean effective gain of antennas in a wireless channel," IET 
Microwaves, Antennas and Propagation, vol. 3, no. 2, pp. 214227, 2009.

[15] P. Corona, G. Ferrara, and M. Migliaccio, "Reverberating chamber electromagnetic field in presence of an unstirred component," IEEE Transactions on Electromagnetic Compatibility, vol. 42, no. 2, pp. 111-115, 2000.

[16] O. Lundén and M. Bäckström, "How to avoid unstirred high frequency components in mode stirred reverberation chambers," in Proceedings of the IEEE International Symposium on Electromagnetic Compatibility (EMC '07), pp. 1-4, Honolulu, Hawaii, USA, July 2007.

[17] R. J. Pirkl, J. M. Ladbury, and K. A. Remley, "The reverberation chamber's unstirred field: a validation of the image theory interpretation," in Proceedings of the International Symposium on Electromagnetic Compatibility (ISEMC '11), pp. 670-675, Long Beach, Calif, USA, August 2011.

[18] B. Clerckx, C. Craeye, D. Vanhoenacker-Janvier, and C. Oestges, "Impact of antenna coupling on $2 \times 2$ MIMO communications," IEEE Transactions on Vehicular Technology, vol. 56, no. 3, pp. 1009-1018, 2007.

[19] I. Szini, CTIA MIMO $2 \times 2$ reference antenna, CTIA Std. MOSG110 904, 2011.

[20] C. A. Balanis, Antenna Theory: Analysis and Design, John Wiley \& Sons, New York, NY, USA, 2nd edition, 1996.

[21] P. J. Smith, L. M. Garth, and S. Loyka, "Exact capacity distribution for MIMO systems with small numbers of antennas," IEEE Communications Letters, vol. 7, no. 10, pp. 481-483, 2003.

[22] C. Fobes, M. Evans, N. Hastings, and B. Peacock, Statistical Distributions, John Wiley \& Sons, 4th edition, 2011.

[23] R. E. Miles, "On random rotations in R3," Biometrika, vol. 52, no. 3, pp. 636-639, 1965.

[24] J. C. Mitchell, "Sampling rotation groups by successive orthogonal images," SIAM Journal on Scientific Computing, vol. 30, no. 1, pp. 525-547, 2007.

[25] A. Yershova, S. Jain, S. M. Lavalle, and J. C. Mitchell, "Generating uniform incremental grids on $\mathrm{SO}(3)$ using the hopf fibration," International Journal of Robotics Research, vol. 29, no. 7, pp. 801-812, 2010. 

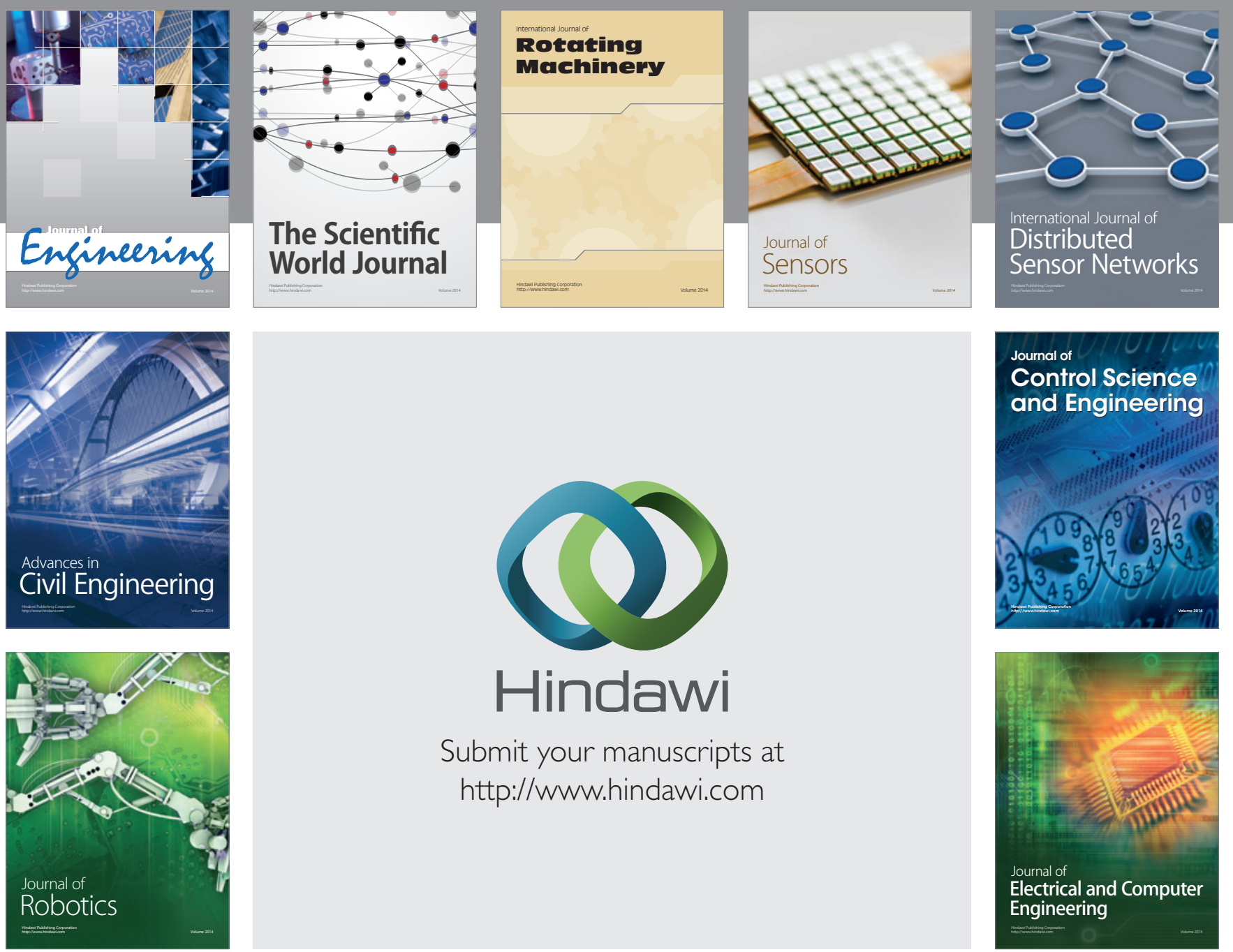

Submit your manuscripts at

http://www.hindawi.com
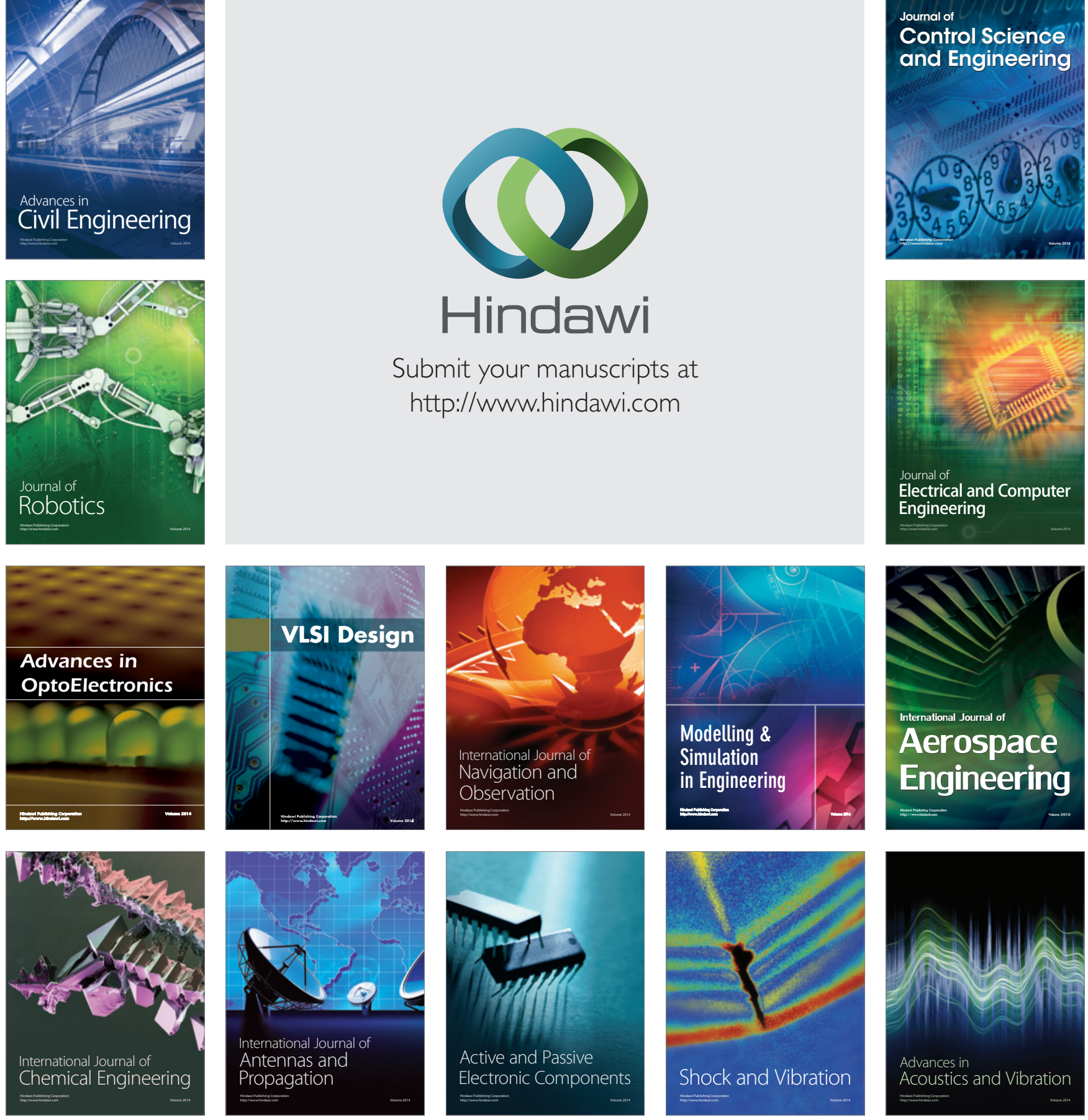